\title{
emm 81, The Predominant Group a Streptococcus from North India in Year 2003 in Context to Adhesion, Invasion and Antimicrobial Susceptibility Pattern
}

\author{
Dapinder Kaur Bakshi ${ }^{1,2}$, Vanita Dhanda1,2, Vivek Sagar ${ }^{1,2}$, Devinder Toor $^{1,2}$, Rajesh Kumar ${ }^{2}$ and Anuradha Chakraborti ${ }^{1 *}$ \\ ${ }^{1}$ Department of Experimental Medicine \& Biotechnology, School of Public Health,Post Graduate Institute of Medical Education \& Research (PGIMER), Chandigarh - \\ 160012, India \\ ${ }^{2}$ Department of Biotechnology, Punjab State Council for Science \& Technology, Chandigarh - 160019, India
}

\begin{abstract}
Heterogeneity exists among Group a Streptococcus emm types worldwide. In 2003, we observed 11 circulating emm types in northern region of India, of which emm 81 was found to be the predominant type (17.5\%). As emm 81 has been reported to be associated with invasive diseases in western countries, hence, in the present study, attempts were made to study the virulence potential of these isolates from skin and throat samples. Isolates were screened for nine fibronectin binding protein (FBP) genes, evaluated for adherence \& invasion potential along with drug resistance to various commonly prescribed antibiotics for treatment. Throat isolates showed higher distribution of FBP genes as compared to skin isolates. All the isolates were found to be positive for $s c i B$ and prtF $15 ; 92.9 \%$ for $s f b$ and $f b a, 78.6 \%$ for sciA; $35.2 \%$ for prtF1; $7.1 \%$ for prtF2 but none for sfb2 and pfbp. Isolates showed low (8.5\%) to moderate $(27.7 \%)$ adherence and negligible invasion potential in the experimental A549 cell line which was confirmed by immunofluorescent confocal microscopy. Drug resistance profiling showed isolates to be highly resistant to macrolides, tetracycline, cotrimaxazole but all susceptible to penicillin. The study shows emm 81 strains from northern India to be of less virulent nature with respect to adherence/invasion potential, highlighting that same emm type in different geographical regions may have a different clinical outcome, the latter being dependent on number of factors like ethnicity, geographical, socioeconomic factors besides its molecular type and source.
\end{abstract}

Keywords: Gas; emm 81; Invasion; Adherence; Fibronectin binding protein; Antibiotic susceptibility

\section{Introduction}

Streptococcus pyogenes, (GAS) is an etiological agent causing wide range of human disorders including pharyngitis, pyoderma and post infection sequela like rheumatic fever/ rheumatic heart disease (RF/RHD). The incidence of such disorders is high in India [1]. M protein, the major virulence antigen of GAS has been found to be highly heterogeneous in India as reported earlier by our laboratory [2]. Beside $\mathrm{M}$ proteins, there are other virulence factors like Streptolysin $0 \&$ S, C5a peptidase, streptococcal protective antigen (spa), streptococcal pyrogenic exotoxin (spe) etc. which have been known to play a key role in pathogenesis of this bacterium. The earlier studies carried out in our laboratory have demonstrated low frequency of spe A gene within Indian isolates which is indicative of less virulent nature of the isolates [3]. However, recently, the presence of virulent streptococcal inhibitor of complement (sic) protein and its encoding genes (closely related sic: crs and distantly related sic: drs) have been documented in Indian isolates [4].

Like other bacteria, the initial step for establishment of streptococcal infection is bacterial adherence and colonization to host tissue for which Streptococcus pyogenes genome encodes multiple adhesin genes for various adherence determinants, out of which fibronectin binding proteins (FBP) are the most important contributors [5]. FBPs like sciA, sciB, sfb,sfb2, prtF1, prtF2,prtF15, $f b a A, f b a B$, $p f b p, s f b I I, f b p-54$ and sof not only enhance the adherence of GAS to epithelial cells but also facilitate the bacteria for invasion and persistence within the host cells, reflecting its virulence [6]. The drug of choice for prevention of GAS infection remains penicillin till date as no penicillin resistant isolate has been reported [7]. Moreover, other drugs like macrolides are being more commonly used for the treatment of GAS infection in penicillin allergic patients. However, there have been worldwide reports of resistance of GAS to commonly used antibiotics with persistent increase in drug resistance $[8,9,10]$.

In the present scenario of changing epidemiology and emergence of new emm types during different seasons and years, it becomes necessary to look into regional prevalence of emm types in a community along with their virulence credentials. However, such data is lacking in Indian context. Apart from this, another challenge being encountered with GAS isolates is increasing resistance towards commonly prescribed antibiotics. Therefore, in the present prospective study the most predominant GAS type obtained from the community during the year 2003 was evaluated for virulence traits in terms of presence of FBP genes, their adherence and invasion potential along with susceptibility to commonly used antibiotics used for their treatment.

\section{Materials and Methods}

Isolation of GAS and emm typing

Approximately 400 children in the age group of seven to eleven

*Corresponding author: Anuradha Chakraborti, Department of Experimenta Medicine and Biotechnology, Post Graduate Institute of Medical Education \& Research (PGIMER), Chandigarh - 160012, India, Tel: +91 172 2746277; Fax: +91 172 2744401, 2745078; E-mail: superoxide@sify.com

Received August 30, 2010; Accepted October 14, 2010; Published October 16 2010

Citation: Bakshi DK, Dhanda V, Sagar V, Toor D, Kumar R, et al. (2010) Review and Analysis of Reported Anthrax-Related Military Mail Security Incidents in Washington D.C. Metropolitan Area During March 2005. J Bacteriol Parasitol 1:103. doi:10.4172/2155-9597.1000103

Copyright: (c) 2010 Bakshi DK, et al. This is an open-access article distributed under the terms of the Creative Commons Attribution License, which permits unrestricted use, distribution, and reproduction in any medium, provided the original author and source are credited. 
Citation: Bakshi DK, Dhanda V, Sagar V, Toor D, Kumar R, et al. (2010) Review and Analysis of Reported Anthrax-Related Military Mail Security Incidents in Washington D.C. Metropolitan Area During March 2005. J Bacteriol Parasitol 1:103. doi:10.4172/2155-9597.1000103

Page 2 of 4

years from different clinics and hospitals in and around urban slum area near Chandigarh, North India with defined GAS disorders like pharyngitis and pyoderma were registered for community survey in the year 2003. Swabs were taken from symptomatic patients by rubbing over both the tonsils and the posterior pharyngeal walls (pharyngitis cases) and skin lesions (pyoderma cases) by a physician after consent from their parents. Throat and skin swabs were streaked on sheep blood agar plates. The plates were screened for beta haemolysis after 24 hours of incubation at $37^{\circ} \mathrm{C}$ in presence of $5 \% \mathrm{CO}_{2}$. The grouping of pure cultures was then done using streptex kit (Murex Biotech, UK). The emm typing of GAS isolates was done using PCR and sequencing $[11,12]$. The emm gene sequence of GAS isolates was subjected to homology search against CDC reference strains (http://www.cdc.gov/ncidod/biotech/strep/strepblast.htm) as well as by BLAST search analysis (http://www.ncbi.nlm.nih.gov/BLAST/ Blast.cgi).

\section{PCR amplification of fibronectin binding protein genes}

Genomic DNA of emm 81 isolates was extracted and amplification of nine sequence specific FBP genes: $s c i A, s c i B, s f b, s f b 2$, prtF1, prtF2, prtF 15 , fba and $p f b p$ were carried out with published primers at standardized conditions [13]. The experiments were repeated twice to confirm the results.

\section{Adherence and invasion assay}

A549 cells (ATCC CCL 185), a human alveolar carcinoma cell line was used to study cell adherence and internalization of group $A$ streptococci, which is a prerequisite for establishment of infection in the host and reflects on its virulence potential. Streptococcal adherence was done by Giemsa staining and further checked by fluorescence activated cell sorter (FACS). Briefly, $100 \mu$ l aliquots of fluorescein isothiocyanate (FITC, Sigma chemicals Co, USA) labeled streptococcal suspension $\left(10^{8}\right.$ bacteria) was added in each well of multi-well plate containing confluent monolayers of cell line $(1: 100)$. After 30 minutes at $37^{\circ} \mathrm{C}$ under $5 \% \mathrm{CO}_{2}$ atmosphere, the wells were washed thrice with $0.15 \mathrm{M} \mathrm{NaCl}$ to remove non-adherent bacteria. The fluorescence associated with adherent streptococci was measured in a flowcytometer (Becton Dickinson, FACS Calibur and USA). Percent adherence was calculated as the number of adherent streptococci, which was calculated from the absolute fluorescence value of the total number of streptococci in each assay. Streptococcal internalization was evaluated by antibiotic protection assay [14]. Immunofluroscent confocal microscopy was also performed [15] for further confirmation. Standard reference strain M1 obtained from ATCC, USA having high adherence and invasion efficiency was run in parallel as control.

\section{Antibiotic sensitivity pattern}

The sensitivity of emm 81 isolates towards the generally prescribed drugs for the GAS treatment was evaluated. Apart from penicillin, the obvious drug of choice for treatment of GAS infection, macrolides including erythromycin and azithromycin as well as other drugs like tetracycline and co-trimaxazole were included in the study due to their wide usage in developing countries. Kirby Bauer disk diffusion method on Muller Hinton agar medium according to National Committee for Clinical Laboratory Standards guidelines for penicillin $\mathrm{G}(10 \mu \mathrm{g})$, co-trimoxazole $(25 \mu \mathrm{g})$, tetracycline $(30 \mu \mathrm{g})$, erythromycin $(15 \mu \mathrm{g})$ and azithromycin $(15 \mu \mathrm{g})$ procured from $\mathrm{BD}$ Biosystem was used [16].

\section{Results}

After confirmation of eighty GAS isolates from throat and skin of children screened in this study, emm type analysis was performed. GAS isolates revealed emm $81(17.5 \%, 14 / 80)$ to be the most predominant type from our region in the year 2003, while frequency of other emm types $11(11.2 \%, 9 / 80), 15(8.7 \%, 7 / 80), 42(7.5 \%, 6 / 80)$, $49(10 \%, 8 / 80), 55(6.2 \%, 5 / 80), 57(7.5 \%, 6 / 80), 66(6.2 \%, 5 / 80), 68(7.5 \%$, 6/80), $103(7.5 \%, 6 / 80)$ and $112(10 \%, 8 / 80)$ (unpublished data) were found to be low. Out of total 14 isolates of emm 81 type, there were seven isolates from patients with pharyngitis \& seven from impetigo. Thirteen isolates were typed as emm 81.1 while a single isolate was typed as emm 81.2. emm 81 being the most predominant type and as reported to be invasive in western countries [16] prompted us to further characterize this type. The PCR analysis of nine FBP genes showed all isolates $(100 \% ; \mathrm{n}=14)$ positive for $s c i B$ and $p r t F 15$ gene, 92.9\% $(\mathrm{n}=13)$ for $s f b \& f b a ; 78.6 \%(\mathrm{n}=11)$ for $s c i A$ genes; $35.2 \%(\mathrm{n}=5)$ for prtF1; $7.1 \%(\mathrm{n}=1)$ for prtF2 and none positive for $s f b 2$ and $p f b p$ gene. All throat isolates were positive for $s c i A, s c i B, s f b, p r t F 15 \& f b a$ genes while $s c i B, s f b$ and prtF15 were present in most of the skin isolates (Table 1).

The adherence pattern of emm 81 isolates to A549 cells was evaluated and the results showed varying degree of binding. Giemsa staining showed low $(1+, \mathrm{n}=9)$ and moderate adherence $(2+$, $\mathrm{n}=5$ ), as compared to standard M1 isolate, a positive control, which showed sufficient adherence to A549 cell (Figure $1 \mathrm{~A}-\mathrm{C}$, Table 1). The qualitative \% adherence calculated by FACS analysis indicated adherence range from $8.5 \%$ to $27.7 \%$. The invasive capacity of emm 81 GAS isolates was evaluated by infecting A549 cells and monitoring the number of viable intracellular bacteria at two, four and twenty hours post infection. To our knowledge, out of the nine genes considered in present study, prtF is known to have well established correlation with invasive potential. No viable count was observed for prtF1 isolates, however low counts were obtained for $p r t F 1^{+}$strains indicating very low cell invasion efficiency which was statistically insignificant. Further immunoflorescent confocal microscopy confirmed moderate adherence with internalization of few GAS isolates to A549 cells.

Further the isolates showed resistance towards all the drugs including tetracycline $(92.9 \%\{\mathrm{R}+\mathrm{I}\}: 78.6 \% \mathrm{R}, 14.28 \% \mathrm{I})$, co-trimoxazole $(78.6 \% R)$, erythromycin $(50 \%\{R+I\}: 14.3 \% R, 35.7 \% \mathrm{I})$ and azithromycin $(64.3 \%\{\mathrm{R}+\mathrm{I}\}: 7.1 \% \mathrm{R}, 57.1 \% \mathrm{I})$. However, no resistance was found for penicillin $\mathrm{G}$. Interestingly, the emm 81.2 isolate from skin was sensitive to both tetracycline and co-trimoxazole whereas most of emm 81.1 strains were resistant.

\section{Discussion}

The heterogeneous emm type prevalence at different time intervals has been reported from India $[2,10]$. However, there is a lack of information regarding invasive/non-invasive nature of these isolates from the region. Our earlier study has shown a low frequency

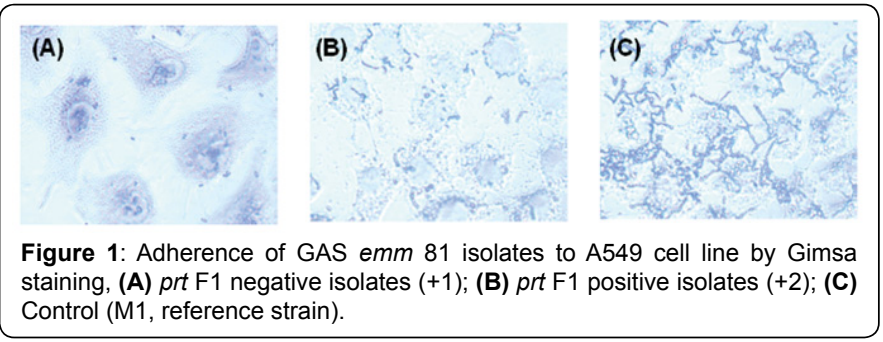


Citation: Bakshi DK, Dhanda V, Sagar V, Toor D, Kumar R, et al. (2010) Review and Analysis of Reported Anthrax-Related Military Mail Security Incidents in Washington D.C. Metropolitan Area During March 2005. J Bacteriol Parasitol 1:103. doi:10.4172/2155-9597.1000103

Page 3 of 4

\begin{tabular}{|c|c|c|c|c|c|c|c|c|c|c|c|c|c|}
\hline \multirow[t]{2}{*}{ Sr. No. } & \multirow[t]{2}{*}{ Source } & \multirow[t]{2}{*}{ Emm type } & \multicolumn{7}{|c|}{ Fibronectin binding protein genes } & \multicolumn{4}{|c|}{ dherence } \\
\hline & & & sci $A$ & sci B & $s f b$ & $s f b 2$ & prtF1 & prtF2 & prtF15 & fba & pfbp & Giemsa & FACS \\
\hline 1 & Throat & 81.1 & + & + & + & - & + & - & + & + & - & $2+$ & $26.70 \%$ \\
\hline 2 & Throat & 81.1 & + & + & + & - & - & - & + & + & - & $1+$ & $13.80 \%$ \\
\hline 3 & Throat & 81.1 & + & + & + & - & - & - & + & + & - & $1+$ & $13.60 \%$ \\
\hline 4 & Throat & 81.1 & + & + & + & - & + & - & + & + & - & $2+$ & $27.73 \%$ \\
\hline 5 & Throat & 81.1 & + & + & + & - & + & - & + & + & - & $2+$ & $23.40 \%$ \\
\hline 6 & Throat & 81.1 & + & + & + & - & - & - & + & + & - & $1+$ & $12.60 \%$ \\
\hline 7 & Throat & 81.1 & + & + & + & - & - & - & + & + & - & $1+$ & $13.40 \%$ \\
\hline 8 & Skin & 81.1 & - & + & + & - & - & - & + & + & - & $1+$ & $17.75 \%$ \\
\hline 9 & Skin & 81.1 & + & + & + & - & - & + & + & - & - & $1+$ & $9.40 \%$ \\
\hline 10 & Skin & 81.1 & + & + & + & - & + & - & + & + & - & $2+$ & $24.40 \%$ \\
\hline 11 & Skin & 81.1 & + & + & + & - & + & - & + & + & - & $2+$ & $26.70 \%$ \\
\hline 12 & Skin & 81.1 & + & + & + & - & - & - & + & + & - & $1+$ & $17.70 \%$ \\
\hline 13 & Skin & 81.1 & - & + & - & - & - & - & + & + & - & $1+$ & $8.50 \%$ \\
\hline 14 & Skin & 81.2 & - & + & + & - & - & - & + & + & - & $1+$ & $22.68 \%$ \\
\hline \multirow[t]{2}{*}{15} & Control & 1 & + & + & - & - & - & - & - & + & - & & \\
\hline & $\%$ & 81 & 78.6 & 100 & 92.9 & 0 & 35.2 & 7.1 & 100 & 92.9 & 0 & & \\
\hline
\end{tabular}

Note: Fbp genes +: presence; - : absence 2+: moderate adherence; 1+: low adherence when compared to standard reference M1 GAS strain

Table 1: Distribution of fibronectin binding protein genes and adherence potential of isolated emm $81 \mathrm{GAS}$ isolates.

of the speA gene, a gene usually associated with scarlet fever or toxic shock-like syndrome, within Indian isolates [3] suggestive of less virulent nature of isolates in Indian community, though the presence of sic genes, a streptococcal virulence factor, has been documented by us in Indian isolates [4].

In the year 2003, the study conducted at our laboratory showed predominance of emm 81 type in North Indian community, which has also been reported from other countries $[8,16,18,19,20]$. Report of association of emm 81 with invasive disease in Sweden and Romania $[17,20]$ prompted us to look for a virulent potential of the circulating predominant emm 81 isolates from our community. We focused on adherence and invasion potential of these isolates towards host cell, the process resulting in infection or disease outcome. FBP genes, the major contributors of adherence/invasion were screened in isolated emm 81 strains, which showed these strains to possess nine known FBP's to a variable extent, indicating their highly heterogeneous nature. The study conducted by Luca-Harari et al [20] explored the presence of only one FBP gene i.e. prtF1 gene in its isolated emm 81 strains and found all the isolates to possess the same. To best of our knowledge, none of the reports in literature has investigated such elaborate distribution of FBP genes among emm 81 GAS isolates, making our study as first of its kind.

As regards adherence-invasion assays, these isolates showed low to moderate adherence. Maximum adherence (22.7- 27.7\%) was observed in $s f b^{+}$and prtF1 $1^{+}$isolates, which decreased to $13.8 \%$ - $17.75 \%$ for $s b^{+}$and prtF1 - isolates, as compared to lowest value of $8.45 \%$ for ones lacking both genes. On the other hand a single strain with $\mathrm{sfb}^{+}$and $\mathrm{prtF2}^{+}$character showed adherence to the value of $9.40 \%$, suggesting the possible role of all these adhesins in adherence potential. The other studies have also suggested that strains possessing two or more of the genes for these FBPs have more adhering capacity as compared to strains possessing none or one of the genes. Further, the negligible/lower internalization efficiency of emm 81, especially prtF1 $1^{+}$isolates may be attributed to lower adherence recorded $(8.5-27.7 \%)$ in spite of presence of $\mathrm{sfb} 1$, prtF1 genes in isolates.

We also looked in for drug susceptibility of these isolates to commonly prescribed drugs in country. No penicillin resistant isolate have ever been reported from any region of world in spite of its extensive and indiscriminate usage [7] and our results also showed susceptibility of all emm81 strains to penicillin. As regards macrolides, insight into Indian literature till 2002 indicated less than $1 \%$ erythromycin resistance rates among GAS which increased to $9.04 \%$ in 2006 [9], while the present study indicated 50\% resistance among emm 81 isolates, comparable to the reports of increasing resistance from other countries of the world $[8,19]$. A new macrolide azithromycin being used extensively in the country for past few years alongwith co-trimaxazole also exhibited high resistance among isolates. Similarly to reports of tetracycline resistance in GAS [9], we found all thirteen emm 81.1 isolates showed resistance to tetracycline while a single isolate, emm 81.2, a skin isolate was susceptible to it. The interesting feature observed was that prtF $1^{+}$ isolates from throat showed resistance to tetracycline, erythromycin and azithromycin but were sensitive to co-trimaxazole, in spite of high resistance shown towards cotrimaxazole by prtF1- isolates. We understand that our study represents only limited number of emm 81 types from north India and the resistance may be only the result of spreading of one or few strains in this limited area. Further work is required with more number of strains to confirm these observations.

The present study reflected predominance of emm 81 during year 2003 in northern region of India with capability of superficial colonization and negligible invasion capacity, an indicative of its less virulent nature as compared to the prevalent GAS isolates reported from other countries. It seems that same emm types in different geographical regions may have a complete different clinical outcome and suggesting that besides the emm type, the disease outcome also depends on ethnicity, geographical and socioeconomic factors.

\section{References}

1. Shet A, Kaplan E (2004) Addressing the burden of group A streptococcal disease in India. Indian J Pediatr 71: 41-48.

2. Nandi S, Ganguly NK, Kumar R, Bakshi DK,Vivek Sagar VS, et al. (2008) Genotyping of group A streptococcus by various molecular methods. Indian J Med Res 127: 71-77.

3. Nandi S, Chakraborti A, Bakshi DK, Rani A, Kumar R, et al. (2002) Association of pyrogenic exotoxin genes with pharyngitis and rheumatic fever/rheumatic heart disease among Indian isolates of Streptococcus pyogenes. Lett Appl Microbiol 35: 237-241. 
Citation: Bakshi DK, Dhanda V, Sagar V, Toor D, Kumar R, et al. (2010) Review and Analysis of Reported Anthrax-Related Military Mail Security Incidents in Washington D.C. Metropolitan Area During March 2005. J Bacteriol Parasitol 1:103. doi:10.4172/2155-9597.1000103

Page 4 of 4

4. Sagar V, Kumar R, Ganguly NK, Chakraborti A (2008) Comparative analysis of emm type pattern of Group A Streptococcus throat and skin isolates from India and their association with closely related SIC, a streptococcal virulence factor. BMC Microbiol 8: 150

5. Hynes W (2004) Virulence factors of the group A streptococci and genes that regulate their expression. Front Biosci 9: 3399-3433.

6. Molinari G, Chhatwal GS (1998) Invasion and survival of Streptococcus pyogenes in eukaryotic cells correlates with the source of the clinical isolates. J Infect Dis 177: 1600-1607.

7. Kaplan EL (1997) Recent evaluation of antimicrobial resistance in betahemolytic streptococci. Clin Infect Dis 24 Suppl 1: S89-S92.

8. Erdem G, Ford J, Johnson D, Abe L, Yamaga K, et al. (2005) Erythromycinresistant group A streptococcal isolates collected between 2000 and 2005 in Oahu, Hawaii and their emm types. J Clin Microbiol 43: 2497-2499.

9. Jacob SE, Lloyd C, Menon T (2006) CMLS and M Phenotypes among Streptococcus pyogenes isolates in Chennai. Indian J Med Microbiol 24: 147148

10. Ray D, Sinha S, Saha S, Karmakar S, Dutta RN, et al. (2010) Preliminary sentinel surveillance report on Antibiotic resistance trend of Streptococcus pyogenes in Kolkata region, India. Al Ameen J Med Sci 3: 146-151.

11. Sagar V, Bakshi DK, Nandi S, Ganguly NK, Kumar R, et al. (2004) Molecular heterogeneity among north Indian isolates of Group A Streptococcus. Let Appl Microbiol 39: 84-88.

12. Beall B, Facklam R, Thompson T (1996) Sequencing emm specific PCR products for routine and accurate typing of group A Streptococcus. J Clin Microbiol 34: 953-958.
13. Vlaminckx BJ, Mascini EM, Schellekens J, Schouls LM, Paauw A, et al. (2003) Site-specific manifestations of invasive group a streptococcal disease type distribution and corresponding patterns of virulence determinants. J Clin Microbiol 41: 4941-4949.

14. Rubens CE, Smith S, Hulse M, Chi EY, Van Belle G (1992) Respiratory epithelial cell invasion by group B streptococci. Infect Immun 60: 5157-5163.

15. Manning PA (1995) Use of confocal microscopy in studying bacterial adhesion and invasion. Methods Enzymol 253: 159-166.

16. Clinical and Laboratory Standards Institute (2009) (CLSI) 2009 performance standards for antimicrobial susceptibility testing. Nineteenth information supplement (M100-S19). Wayne, PA: Clinical and Laboratory Standards Institute.

17. Darenberg J, Luca-Harari B, Jasir A, Sandgren A, Pettersson H, et al. (2007) Molecular and clinical characteristics of invasive group A streptococcal infection in Sweden. Clin Infect Dis 45: 450-458.

18. Facklam R, Beall B, Efstratiou A, Fischetti V, Johnson D, et al. (1999) emm typing and validation of provisional $\mathrm{M}$ types for Group A Streptococci. Emerg Infect Dis 5: 247-253.

19. Ho PL, Johnson DR, Yue AW, Tsang DN, Que TL, et al. (2003) Epidemiological analysis of invasive and noninvasive group A streptococcal isolates in Hong kong. J Clin Microbial 41: 937-942.

20. Luca-Harari B, Straut M, Cretoiu S, Surdeanu M, Ungureanu V, et al. (2008) Molecular characterization of invasive and non-invasive Streptococcus pyogenes isolates from Romania. J Med Microbiol 57: 1354-1363. 